\title{
USO Y ABUSOS DEL DERECHO A MORIR*
}

\author{
Diana Cohen Agrest** \\ Universidad de Buenos Aires, Argentina \\ cohendiana@gmail.com
}

\begin{abstract}
RESUMEN: Tradicionalmente, la Doctrina de la Santidad de la vida humana reguló las conductas del fin de la vida, independientemente de que se fundara su sacralidad o bien en un Dios personal o bien en el valor que le atribuimos a la vida per se, siendo éste el punto crucial en el que se centra la prohibición de intervenir en prácticas eutanásicas y de muerte asistida. Sin embargo, por primera vez en la historia humana, intervenciones invasivas condensadas en la expresión "encarnizamiento terapéutico" permiten sostener una vida meramente biológica, sin posibilidades de desplegar una vida biográfica. Confrontado a esa perspectiva amenazante, el ser humano puede tomar la decisión de morir. Cuando esa conducta condensa actitudes autónomas, el Estado no puede obligar a seguir la doctrina de la Santidad de la vida humana, violando el principio de libertad de conciencia. El escenario es diferente cuando se discute la posibilidad de regular, mediante políticas públicas, las prácticas eutanásicas y de suicidio asistido. En esos escenarios, el fantasma del abuso por parte de la familia, del personal sanitario y de las instituciones (hospital, seguridad social, etc.) amenaza las mejores intenciones. Se trata, entonces, de examinar el peso de esas amenazas en los diversos contextos socioculturales.
\end{abstract}

Palabras clave: Muerte digna, eutanasia, autonomia, abusos.

\section{USE AND ABUSES OF THE RIGHT TO DIE}

ABSTRACT: Traditionally, the Doctrine of the Sanctity of Human Life has regulated practices associated to the end of life decisions. That is, indeed, the main argument of the prohibition of acts of euthanasia and assisted death. However, the emergence

Trabajo recibido el 30 de agosto y aprobado el 17 de octubre de 2011.

** Doctora en Filosofía por la Universidad de Buenos Aires y magíster en Bioética por el Centre for Human Bioethics de la Monash University de Australia. Docente e investigadora en el Departamento de Filosofía de la Facultad de Filosofía y Letras y en la Facultad de Medicina de la Universidad de Buenos Aires. 
of new biomedical technologies created a disruption in that millenary order by using invasive procedures that allow merely biological life support, but making unable to deploy a biographical life. Faced with that threatening prospect, the human being can decide to die. When such conduct involves autonomous attitudes it assumes a hardly disputable value, since the State cannot force its citizens to follow a certain doctrine, as is the Sanctity of Human Life, without violating the principle of freedom of conscience, particularly when it comes to idiosyncratic decisions concerning the private individual sphere. The scenario is different when what is in discussion is the possibility of regulating the practices of euthanasia and assisted suicide through public policies: the ghost of abuse perpetuated by the family, medical personnel and institutions (hospitals, social security, etc.) threatens even the best intentions. The weight of those threats, however, differs according to various socio-cultural contexts.

Keywords: Death with dignity, euthanasia, autonomy, abuses.

\section{INTRODUCCIÓN: LA DOCTRINA DE LA SANTIDAD DE LA VIDA}

La Doctrina de la Santidad de la vida humana -asociada al mandamiento que ordena no matar-, guió las conductas del fin de la vida. Según sus premisas, si la vida es un bien dado al hombre por su Creador, es Dios quien nos la concede y es Dios quien nos la quita. Lo cierto es que casi todos compartimos la idea de que la vida humana individual es sagrada, independientemente de que fundemos su sacralidad en un Dios personal, tal como se sostuvo esta creencia desde tiempos inmemoriales o bien en el valor que le atribuimos a la vida per se. Pues pese a que desde siempre se ha concebido la sacralidad como un concepto asociado al culto religioso, en otra de sus acepciones contemplada por el Diccionario de la Real Academia, lo sacro es definido como aquello "digno de veneración y respeto". Siguiendo esta línea conceptual, se interpreta el término "sagrado" en un sentido no necesariamente teísta. Desde esta perspectiva secular, reconocemos en lo sagrado un valor intrínseco; toda vez que lamentamos la destrucción de una obra de arte o la extinción de una especie botánica o zoológica en la naturaleza es porque reconocemos en todas ellas dicho valor, le atribuyamos o no cierto origen trascendente ${ }^{1}$. En este sentido, la vida humana es sagrada en virtud del valor que le atribuimos a la vida como tal.

La santidad de la vida humana -religiosa o secular- fue el argumento nuclear en el que se centró la prohibición de intervenir en las prácticas asociadas a la denominada "muerte digna”. Pero es notorio que la aparición de nuevas tecnologías biomédicas irrumpió en este orden milenario aún vigente en cualquiera de sus versiones, la religiosa y/o la secular, pues por primera vez en la historia humana, las intervenciones invasivas condensadas en la expresión "encarnizamiento terapéutico" constituyen un instrumento perverso invocado para sostener la san-

Dworkin, Ronald. El dominio de la vida, Barcelona, España: Editorial Ariel, 1998, p. 100. 
tidad de la vida a costa de una pieza sacrificial: un ser humano que sufre cuando, antes de los progresos biotecnológicos, habría abandonado este mundo, -expresándolo incluso en la retórica religiosa- "cuando Dios lo dispusiera". Con el propósito de desalentar dicha práctica, en los últimos tiempos se alega, una y otra vez, que los cuidados paliativos constituyen el instrumento éticamente idóneo para morir con dignidad.

\section{LA AUTONOMÍA DEL PACIENTE FRENTE A LAS MODALIDADES DE ABUSO}

\section{I. ENCARNIZAMIENTO TERAPÉUtico: LA VIDA COMO AMENAZA}

Se invocan, a menudo, la posibilidad de aplicar cuidados paliativos, caracterizados en la definición de 2002 de la OMS, como el " modo de abordar la enfermedad avanzada e incurable que pretende mejorar la calidad de vida tanto de los pacientes que afrontan una enfermedad como de sus familias, mediante la prevención y el alivio del sufrimiento a través de un diagnóstico precoz, una evaluación adecuada y el oportuno tratamiento del dolor y de otros problemas tanto físicos como psicosociales y espirituales". Presuntamente, los cuidados paliativos deberían formar parte de la atención sanitaria ordinaria para todos los pacientes gravemente enfermos, aunque numerosos estudios probaron una distancia significativa entre el abanico de posibilidades inauguradas por dichos tratamientos y la práctica real, pues suelen ser provistos por contados nosocomios y su cobertura por la prepaga o la obra social suele alcanzar a escasas prestaciones.

Pero lo cierto es que aun cuando se reciban tratamientos paliativos de excelencia, de hecho ciertos síntomas son intratables y, por añadidura, al sufrimiento físico se le suman las dimensiones psicológicas, sociales, existenciales que deben ser asimismo contempladas. Anticipándose a una horrible agonía, pueden invocar el derecho a rechazar un tratamiento o a someterse a una práctica eutanásica o a una muerte asistida por un médico: se trata, al fin de cuentas, de evitar aquel "encarnizamiento terapéutico", que no es sino una forma de obstinación irracional. Los pacientes terminales, observadores impotentes de su deterioro irreversible, a menudo suelen ver la muerte como la única vía de escape a su sufrimiento y hasta como el único medio de autopreservar la identidad de quienes fueron alguna vez.

Desde el punto de vista profesional es posible reconocer que, en ciertos estadios, la vida humana tiene una calidad tan deteriorada que no se actúa en beneficio del enfermo cuando se lo mantiene con vida a toda costa. Algunos estiman que la eutanasia pasiva, que consiste en omitir hacer, es éticamente permisible, mientras que la eutanasia activa, que consiste en un hacer, no lo es. De acuerdo con esta creencia, abstenerse de reanimar a un enfermo en fase terminal que presenta una falla cardíaca tras otra, es realizar un acto eutanásico pasivo, y en este caso la responsabilidad moral del médico no debe ser puesta en tela de juicio. En cambio, inyectar una dosis letal de potasio a un paciente incurable es una eutanasia activa, y allí sí se juega la 
responsabilidad moral del médico. Sin embargo, justificar filosóficamente semejante actitud es, sin lugar a dudas, más difícil de lo que podría parecer inicialmente. Porque pese a que una de nuestras intuiciones morales más básicas se resume en la premisa de que somos responsables de nuestras acciones pero no lo somos en el mismo grado de nuestras omisiones, dicha creencia es inconsistente, tal como lo reconoce la filosofía de la acción y el derecho penal en cuyo marco la distinción entre actuar por omisión y por comisión está tipificada. ${ }^{2}$ Estas distinciones conceptuales en pugna expresan el entramado en el que se invoca el derecho a decidir sobre el propio cuerpo como un derecho humano fundamental que a menudo colisiona con las premisas de un Estado paternalista.

\subsection{El Derecho a morir como derecho humano fundamental VERSUS el Estado PATERNALISTA}

Aunque los valores en juego son el derecho moral y legal a decidir sobre el destino del propio cuerpo y de la propia vida, las objeciones invocadas a las prácticas eutanásicas y de ayuda al morir (carácter sagrado de la vida, mantenimiento de la integridad de la profesión médica) son paternalistas, pues pretenden justificar en nombre de una concepción particular de lo que se estima como una vida buena, lo que no es sino un enmascaramiento de una intrusión en una decisión cuyo autor debería ser el individuo afectado. Correlativamente, por su índole indeclinable, el Estado no puede imponer determinada doctrina, como la de la Santidad de la vida humana, violando el principio de libertad de conciencia, en particular cuando se trata de decisiones personalísimas que conciernen a la esfera privada. ${ }^{3}$

Si contemplamos el derecho a una muerte digna, entendido como un derecho humano fundamental que colisiona con las imposiciones de un Estado paternalista, se ha de tomar en cuenta que el hecho de que una decisión parezca ir a contracorriente del bienestar de un individuo no aporta las razones éticas ni el fundamento legal para rechazar su petición. Si la decisión pudiese ser simplemente ignorada cuando, desde la perspectiva de terceros, daña el bienestar de la persona, ¿qué sentido tendría, entonces, la autonomía tan proclamada cuando no pasa de ser una retórica vacía? Si es exigible un criterio heterónomo para legitimar una decisión autónoma, ¿de qué autonomía, pues, hablamos?

2 Pero lo cierto es que en uno y otro caso, la consecuencia es la misma. Muchos piensan que la prohibición de la eutanasia activa que corre paralela a la permisibilidad de la pasiva, encubre una actitud tan conservadora como hipócrita, a tal punto que muchos se niegan a considerar que esta modalidad de eutanasia pasiva sea, etimológicamente interpretada "eu-tanasia", una buena muerte. Obviamente, el debate suscitado por esta cuestión es inabarcable en estas páginas. Véase, entre otros, Foot, Philippa. "Killing and Letting Die". En: Steinbock, Bonnie y Norcross, Alastair (eds.). Killing and Letting Die -Second Edition-, Nueva York, Estados Unidos: Fordham University Press, 1994, pp. 280-289; Rachels, James, "Active and Passive Euthanasia", New England Journal of Medicine 292 (enero 9, 1975), pp. 78-80; Brody, Baruch, "Withdrawal of Treatment versus Killing of Patients". En: Beauchamp, Tom L. (ed.), Intending Death: The Ethics of Assisted Suicide and Euthanasia, Nueva Jersey, Estados Unidos: Prentice Hall, 1996.

3 Véase Dworkin, Ronald, op. cit. (n.1), pp. 93-135. 
Así pues, una vez admitida la premisa de la vigencia del respeto al derecho a la autonomía, cuando un paciente terminal competente toma la decisión de poner fin a su vida mediante un consentimiento informado, libre y voluntario, si somos consistentes debemos concluir la licitud moral no sólo del rechazo de tratamiento sino también de las prácticas eutanásicas y la muerte asistida por un médico (o "suicidio asistido", expresión ésta que está siendo paulatinamente abandonada debido a la discutible connotación de perturbación mental e irracionalidad asociados a la noción de suicidio). Cuando esa conducta condensa actitudes autónomas, adquiere un valor difícilmente discutible. Pues ante la alternativa de una vida meramente biológica, sin posibilidades de desplegar una vida biográfica y desprovista de un mínimo contenido existencial, paradójicamente, la muerte voluntaria puede estar signada, precisamente, por su racionalidad. Pero aun cuando su sistema de creencias admitan estas premisas, los profesionales temen involucrarse en estas prácticas, temerosos de un eventual juicio de mala praxis.

\subsection{El problema de la Pendiente resbaladiza}

Aunque ayudar a morir sigue siendo un acto de desobediencia civil, numerosos médicos admiten haber violado la ley, desafiando los sistemas profesionales y legales: las encuestas prueban que, en la intimidad del encuentro clínico, la muerte asistida por el médico es una práctica oculta que suele ser aceptada siempre y cuando no se dé publicidad a la misma ("No preguntes, no digas" ${ }^{4} .^{5}$

Sin embargo, el escenario se complejiza toda vez que se discute la posibilidad de regular, mediante políticas públicas, las prácticas eutanásicas y la muerte asistida por un médico. Por una parte, la mayoría de la gente reconoce casos de sufrimiento severo que no se puede aliviar con cuidados paliativos eficientes. Por otra parte, hay temores bien fundados de que pueda ser un recurso expeditivo para eliminar el sufrimiento de pacientes vulnerables, excusando el intento de suministrar los cuidados paliativos apropiados. ${ }^{6}$

4 Quill, Timothy E. y Greenlaw, Jane, Physician Assisted Death en Bioethics Briefing Book <en línea> http://www.thehastingscenter.org/Publications/BriefingBook/Detail.aspx?id=2202.

5 Un sondeo sobre eutanasia y muerte asistida publicado en 2002 por la revista Oncología clínica -donde sobre 120 encuestas se obtuvo un retorno del 63,3 por ciento-, arrojó los siguientes guarismos: el 26 por ciento de los encuestados aplicó una o más veces estos procedimientos (de ellos, un 45 por ciento dijo que a pedido del paciente y en el resto la iniciativa surgió de la familia o del médico) y un 63\% (48/76) opinó que la eutanasia estaría justificada por algún motivo y, de ser legalizada, estaría dispuesto a realizarla mientras que un 37\% (28/76) opinó que no. Estos índices confirmaron los resultados de un sondeo realizado un ańo antes en Buenos Aires y Gran Buenos Aires que arrojó que el 55 por ciento de los consultados aprobaba la eutanasia y el 56 por ciento que la aceptaría aun para un familiar cercano (Macke, D., Park, W., Rodrigo, J. y Vinocur, D, en: Oncología Clínica; vol. 7 - No 1 - Abril 2002).

6 Los defensores del respeto a ultranza del código hipocrático nos recuerdan que los médicos y enfermeros se deben consagrar a la preservación de la vida. La eutanasia y la muerte asistida, observan, alterarían este compromiso milenario. Y hasta ejercerían una influencia negativa, puesto que podrían dar lugar a que los profesionales no se esfuercen lo suficiente para salvar la vida de un paciente en estado de gravedad. Podrían decidir que el paciente, sencillamente, "estaría mejor muerto" y dar los pasos necesarios para que ello suceda. Esta actitud podría trasladarse hacia pacientes enfermos de menor gravedad. El resultado sería 
El argumento conocido con el nombre de la pendiente resbaladiza sostiene que sólo hay un paso desde la eutanasia voluntaria o la muerte asistida a la eutanasia dirigida administrada a un paciente que no ha dado su consentimiento. Y de allí, sólo otro paso mediará para imponer la eutanasia en contra de los deseos del paciente, ejercida como parte de una política social. Con el propósito de evitar dicha pendiente, se han propuesto protocolos que contemplan la evaluación psicológica y el asesoramiento al paciente, junto con un período prudencial entre la solicitación y la efectivización del acto.

Mediante una evaluación psicológica diseñada para proteger al paciente, se procura identificar psicopatologías o perturbaciones psíquicas que pueden afectar la capacidad de alcanzar una decisión autónoma y voluntaria. Sin embargo, no es un fundamento suficiente de que la detección de una depresión constituya una condición suficiente para rechazar una solicitud de eutanasia o de muerte asistida, la depresión es un factor natural en las enfermedades terminales. En todo caso se puede emplear para identificar casos de depresiones definitivas, irreversibles de depresiones transitorias y reversibles.

En cuanto al asesoramiento profesional, a diferencia, por ejemplo, de los riesgos en adolescentes con ideaciones suicidas que requieren la intervención directiva del profesional, en los casos de enfermos terminales o de discapacitados graves irreversibles, el asesoramiento debe ser no directivo, neutral, diseñado para ayudar al paciente a descubrir si su pedido es genuino, reflexivo, y coherente con sus valores más básicos, si es la elección correcta o la mejor para esa persona. Asegurándose, mediante medidas precautorias apropiadas, que no se trate de un grito de ayuda o el producto de manipulación externa o de cualquier otra modalidad de abuso. Por último, con el propósito de asegurarse de que la elección es estable y duradera, más que una respuesta transitoria o un acting out, se ha propuesto el pedido reiterado o cierto tiempo de espera desde la solicitud hasta la efectivización. Asimismo, un dispositivo de protección que se debe hacer a un lado son las deliberaciones en un comité que protegerá a la institución más que al paciente. Otros mecanismos privilegiados son los testamentos vitales redactados con anterioridad.

Sin embargo, cuando se discute la legalización de estas prácticas, los protocolos parecen no ser suficientes. En los Estados Unidos, la mayoría de las encuestas concluyen que aproximadamente dos tercios de la población aprueba la muerte asistida por un médico como una opción legítima para pacientes terminales con sufrimiento no paliable. Pero cuando se debe optar por la legalización, ese porcentaje desciende al 50 por ciento, brecha que refleja la tensión inherente al debate. Adviértase que la cuestión de si se caerá en un abuso compete a las políticas sociales y públicas, no a los actos de los particulares: no importa tanto si se pueden dar casos aislados, marginales de abuso cada tanto sino si la legislación permite o promueve esos casos, eso es, si la legalización de la eutanasia activa o la muerte asistida promueve un modelo abusivo

una declinación general de la calidad de la atención médica (véase Cohen Agrest, Diana, Por mano propia: estudio sobre las prácticas suicidas, Buenos Aires, Argentina: Fondo de Cultura Económica, 2007: 265-266). 
de dichas prácticas. Sin embargo, pese a las medidas precautorias recién mencionadas, en ciertos escenarios el fantasma del abuso por parte de la familia, del personal sanitario y de las instituciones (hospital, seguridad social, etc.) amenaza las mejores intenciones. Siguiendo las propuestas de Margaret P. Battin ${ }^{7}$, examinaré si el abuso puede ser impedido y cuáles son las estrategias precautorias que desalienten el ejercicio de dichos abusos.

\subsection{Abuso interpersonal}

El más esperable es el de situaciones intrafamiliares, cuyas presiones pueden ser el producto de años de hostilidad o de cierto cansancio emocional de los cuidadores familiares a cargo, tantas veces experimentado por quienes cuidan de un paciente que sufre una enfermedad terminal deteriorante con una agonía prolongada. La vida familiar no siempre es armoniosa y las patologías subyacentes son exacerbadas por el estrés provocado por una enfermedad terminal. Procurando, presuntamente, el alivio del paciente, no siempre reconocen que es su propio alivio el que se juega en su deseo de apurar el desenlace. Los mensajes manipuladores familiares pueden ser explícitos o implícitos, y el espectro de su tonalidad afectiva puede ir desde el ruego o el elogio hasta el empleo de tácticas tales como amenazas y mentiras.

\subsection{Abuso profesional}

El abuso profesional -que consiste en inducir al paciente a solicitar un acto eutanásico presuntamente voluntario- puede presentar la mayoría de los rasgos que caracterizan al abuso interpersonal familiar: sugestión, manipulación y amenazas. Pero a estas tácticas se suma el peso de la autoridad profesional que puede dar lugar a modalidades extorsivas. Si los familiares pueden manipular al enfermo -vulnerables por el dolor, ansiedad, temor de perder los vínculos afectivos y temor a la muerte-, mucho más lo pueden hacer los médicos con una variedad de estrategias. Por ejemplo, pueden emitir diagnósticos imprecisos o pronósticos poco confiables, atemorizar a los pacientes prediciéndoles dolores insoportables, declinar ofrecer control del dolor amparados en el riesgo de adicción a los narcóticos o pueden incluso ofrecer solo un control del dolor esporádico o con efectos colaterales indeseables o, lisa y llanamente, no ofrecer tratamientos que aliviarían los síntomas. Y hasta pueden sugerir al paciente o a la familia que la eutanasia o la muerte asistida es la solución a todos los problemas que no pueden resolver o, por qué no, hasta para ocultar sus errores médicos.

La autoridad profesional condiciona, asimismo, la elección del paciente porque la interacción entre el médico y el paciente es deudora de la confianza depositada en el profesional a través del consentimiento informado. Pero dicha confianza también refuerza la diferencia de poder entre ambos, exacerbando a veces el poder de la autoridad profesional: el médico es quien

Battin, Margaret Pabst, "Voluntary Euthanasia and the Risk of Abuse", The Least Worst Death, Nueva York, Estados Unidos: Oxford University Press, 1994, pp. 163-180. 
identifica el problema propone las alternativas de tratamientos y controla cuántas propone al paciente. Pero no se trata de un valor entre otros, se trata ni más ni menos de si la persona prefiere una vida prolongada pese al dolor o al sufrimiento, o si prefiere un fin más temprano y fácil evitando así el dolor o el sufrimiento. Si el consentimiento a estas prácticas se trata del mismo modo en que se tratan otros procedimientos médicos, lo que está en juego es la agenda del médico y no la del paciente, y el paciente solo puede aceptar o rechazar la propuesta, lo que da lugar al abuso.

Otra de las modalidades abusivas es el paternalismo motivado por cierto temor de ser acusados de mala praxis por error médico. Pero también puede haber médicos bien intencionados que buscan influir en el paciente en su beneficio. La manipulación del paciente cuando el médico percibe que actúa en beneficio del paciente depende de la naturaleza del paternalismo y de si el abuso se define como una violación de una elección voluntaria o de la violación de los intereses del paciente. Una forma de proteger del abuso es la exigencia de documentación de cualquier procedimiento eutanásico o de asistencia para morir. Esa documentación debe formar parte de la historia clínica, y debe incluir el pronóstico, la naturaleza del problema actual, las razones de la solicitud del paciente (tanto de las mencionadas por el paciente como las percibidas por el médico) y un registro de las conversaciones del médico con la familia, si las hubo. También debe registrarse un pedido activo - una carta, una grabación de la voz del paciente y de testigos. Lo importante es que se documente que el pedido surge del paciente y no de la familia o del médico o de la institución.

\subsection{Abuso institucional}

En contraste con el abuso profesional, en el caso de las políticas institucionales -aunque son abiertas y ampliamente conocidas-, pueden acarrear consecuencias coercitivas o de manipulación. Frente a quienes sostienen que es no solo éticamente permisible, sino hasta obligatorio el negar recursos si la proporción costo-beneficio no lo justifica, en especial cuando son solventado por el erario público, el riesgo de abuso institucional se incrementa por el mero hecho de que una parte importante de los gastos en salud se gastan en los últimos meses de vida: cuanto más breve y fácil sea el período terminal, más económica es la cobertura, proporción que puede ser una invitación al abuso institucional. Los temores suelen orientarse hacia la posibilidad de que los hospitales no ofrezcan cierto tipo de atención o de que la ofrezcan a unos pocos pacientes, que los geriátricos permitan que la calidad de su atención se deteriore o que las compañías de seguro excluyan de la cobertura tratamientos y medidas paliativas que pueden beneficiar al paciente. Los críticos de las prácticas eutanásicas y de la muerte asistida suelen alegar que una prueba actual, real, de estas predicciones, son las presiones ejercidas por la seguridad social o la medicina prepaga, confrontadas con los ingentes costos económicos de los enfermos crónicos o en estado terminal. 
Frente a esta advertencia pragmática, se suele replicar que es posible diseñar y hacer cumplir mecanismos de control que impidan el abuso de médicos e instituciones de salud y, a través de regulaciones y contralores, se evite la presunta caída hacia la temible pendiente. Dichos mecanismos en la atención de un enfermo terminal pueden ir desde el control institucional (en hospitales, geriátricos compañías de seguros, agencias gubernamentales), hasta la prohibición de honorarios, de publicidad y de todas aquellas medidas tendientes a alentar el desarrollo de un mercado eutanásico.

La revisión retrospectiva y objetiva de los datos suministrados por el médico a cargo a la institución de salud responsable y de ésta a una agencia pública que sirva de contralor, debería incluir el registro de la enfermedad terminal, el tiempo de relación entre el médico y el paciente, los cuidados paliativos suministrados, el grado de discapacidad, edad, género, etc. Estos datos colaborarían en la detección de aquellos profesionales o instituciones que estadísticamente sobresalen por el número de intervenciones. Si ese número superara la media registrada, se podría sospechar negligencia médica o el rechazo de suministrar medidas analgésicas apropiadas para un control efectivo del dolor.

\section{CONCLUSIÓN: ¿DERECHO O DEBER?}

Cuando son solicitados autónomamente, ningún argumento moral secular prueba que las prácticas de la eutanasia voluntaria y del suicidio asistido son moralmente incorrectas. Y si bien la ética es independiente de la ley, las leyes se hallan sujetas a una evaluación desde una perspectiva ética. En el debate público es común mencionar, a modo de clausura argumentativa, las legislaciones vigentes acerca de una determinada práctica. Sin embargo, toda vez que se examina la legitimidad o ilegitimidad moral de la eutanasia o la muerte asistida, el hecho de que algunas legislaciones las regulen y que otras las prohíban no hace a la moralidad o inmoralidad de esas prácticas. El orden es precisamente el inverso: se mencionan las fuentes jurídicas sólo para descubrir allí los valores éticos que son los pilares de la juridicidad, tal como lo es el valor de la vida confrontado en estas circunstancias con el valor de la autonomía.

Ciertamente, ese debería ser el orden. Y quizá lo sea en determinados países con una cultura de respeto hacia la ley que, en la mayoría de las sociedades, de hecho se encuentra ausente. Pues cuando lo que se halla en juego es la vida de personas tan vulnerables, ¿qué control se puede esperar ejercer en aquellas sociedades con escaso acatamiento del orden jurídico, donde ni siquiera se respetan los derechos morales y legales de las personas con pleno uso de sus facultades físicas y psíquicas? En determinados contextos signados por las violaciones de las normas, el ordenamiento jurídico de estas prácticas no garantiza necesariamente una elección libre y genuina de la persona sobre las que recaen estas prácticas.

De allí que en las sociedades plurales que privilegian el derecho a la autodeterminación, el debate no se centra tanto en la moralidad o inmoralidad de estas prácticas, sino más bien en 
las medidas preventivas para regularlas. Su normativización debería prever e impedir todo riesgo de abuso de la familia, el hospital o sanatorio y hasta de la prepaga u obra social, cada una de cuyas partes podría tener cierto interés en que el "derecho a morir" se convirtiera en un "deber de morir". En estas páginas, pasamos revista a algunas estrategias preventivas del abuso por parte de los distintos agentes y niveles de la atención sanitaria.

En la República Argentina, vienen debatiéndose en las cámaras legislativas distintos proyectos con el fin de alcanzar una normativa que regule la muerte digna. Más allá de los distintos matices que distinguen las propuestas, la legalización debería disipar el fantasma de los juicios por mala praxis en los profesionales -garantizando la toma de decisión en el sistema de salud, promoviendo incluso la consulta a los comités de ética institucionales y evitando la judicialización de los casos-. Alentando que, en el marco del ordenamiento jurídico, la voluntad genuina del paciente o de la familia -en casos de neonatos o pacientes en estado vegetativo irreversible-, guíe el proceder del profesional en la intimidad del encuentro clínico.

\section{REFERENCIAS BIBLIOGRÁFICAS}

Battin, Margaret Pabst, "Voluntary Euthansia and the Risk of abuse". En: The Least Worst Death, Nueva York, Estados Unidos: Oxford University Press, 1994, pp. 163-180.

Cohen Agrest, Diana, Por mano propia: estudio sobre las prácticas suicidas, Buenos Aires, Argentina: Fondo de Cultura Económica, 2007: 265-266.

Dworkin, Ronald, El dominio de la vida, Barcelona, España: Editorial Ariel, 1998.

Feinberg, Joel, «Whose Life is Anyway?». En: Ethical Issues in Death and Dying -Second edition- Tom Beauchamp y Robert Veatch (eds.), Nueva Jersey, Estados Unidos: Prentice Hall, 1996, pp. 138-143.

Macke, D., Park, W., Rodrigo, J., Vinocur, D., Eutanasia y suicidio asistido: encuesta en médicos del CEMIC, en: Revista de Oncología Clínica, vol. 7 - No 1 - Abril 2002, pp. 708-713.

Quill, Timothy E. y Greenlaw, Jane, Physician Assisted Death en Bioethics Briefing Book. <En línea> The Hastings center http://www.thehastingscenter.org/Publications/BriefingBook/Detail.aspx?id=2202. 